\title{
Reduced kidney function is associated with BMD, bone loss and markers of mineral homeostasis in older women: a 10-year longitudinal study
}

\author{
L. Malmgren $^{1,2}$ - F. McGuigan ${ }^{1,2}$ - A. Christensson ${ }^{1,3}$ - K. E. Akesson ${ }^{1,2}$
}

Received: 7 April 2017 / Accepted: 6 September 2017 / Published online: 16 October 2017

(C) The Author(s) 2017. This article is an open access publication

\begin{abstract}
Summary Kidney function decreases with age; however, the long-term influence on bone density (BMD) in older women already at risk of osteoporosis is unknown. We followed kidney function and bone loss for 10 years. Declining kidney function was adversely associated with bone loss and mineral homeostasis in old women, though it attenuated with advanced aging.

Introduction Existing studies do not fully address the relationship between kidney function and bone metabolism with advanced aging in Caucasian women. This study describes the association between kidney function, BMD, bone loss and bone metabolism in older women and provides a review of the available literature for context.

Methods We studied participants from the OPRA cohort with follow-up after 5 and 10 years. Using plasma cystatin $\mathrm{C}$ (cysC), estimated glomerular function rate (eGFR) was evaluated at age $75(n=981), 80(n=685)$ and $85(n=365)$. Women were stratified into "normal" function (CKD stages 1-2), "intermediate" (stage 3a) and "poor" (stages 3b-5), and
\end{abstract}

Electronic supplementary material The online version of this article (https://doi.org/10.1007/s00198-017-4221-y) contains supplementary material, which is available to authorized users.

K. E. Akesson

kristina.akesson@med.lu.se

1 Department of Clinical Sciences Malmö, Clinical and Molecular Osteoporosis Research Unit, Lund University, 214

28 Malmö, Sweden

2 Department of Orthopedics, Skåne University Hospital, 214 28 Malmö, Sweden

3 Department of Nephrology, Skåne University Hospital, S-205 02 Malmö, Sweden outcome measures-BMD, bone loss and markers of mineral homeostasis-were compared.

Results Femoral neck (FN) BMD positively associated with kidney function at 75 years old $(\hat{\beta}=0.001, p=0.028)$ and 80 years old $(\hat{\beta}=0.001, p=0.001)$, although with small effect size. Prevalence of osteoporosis (FN T-score $\leq-2.5)$ did not differ with kidney function. Measured at age 75 , women with poor kidney function had higher annual percentage bone loss over 5 years compared to those with normal function $(2.3 \%$, 95\% CI $1.8-2.8$ versus $1.3 \%, 95 \%$ CI $1.1-1.5, p=0.007$ ), although not when measured from age 80 or 85 . Additionally, markers of mineral homeostasis (PTH, phosphate, vitamin D, calcium), CRP and osteocalcin differed by kidney function. Conclusions In old women, kidney function is associated with BMD, bone loss and altered mineral homeostasis; probably, a relationship attenuated in the very elderly.

Keywords BMD · eGFR · Elderly $\cdot$ Kidney disease $\cdot$ Mineral homeostasis · Women

\section{Introduction}

In the coming years, we face a sizeable increase in the older population [1], and consequently, the prevalence of many agerelated conditions will increase. This includes osteoporosis and, also of importance for bone health, reduced kidney function and chronic kidney disease (CKD) [2, 3]. Chronic kidney disease [4] can lead to disturbed mineral homeostasis, increasing or decreasing the circulating levels of calcium, phosphorus, FGF-23, vitamin D and parathyroid hormone. This effect on mineral homeostasis may be associated with increased bone fragility [5]. 
It is yet to be determined, however, how reduced kidney function affects bone health in a "normal" population, not diagnosed with CKD. We have previously shown that close to $95 \%$ of women after the age of 75 have a mild to moderate reduction of kidney function (CKD stages 2-3), with an accelerated loss between ages 80 and 85 [6]. This indicates a potentially large population at risk, in the event that even mild-tomoderate reduction of kidney function has a negative impact on bone health in older women. To correctly estimate kidney function in the elderly is challenging: glomerular filtration rate can be estimated (eGFR) using either plasma creatinine or cystatin $\mathrm{C}$ (cysC) [7]. However, both have drawbacks; diet and muscle mass affect creatinine values, while cysC levels can be affected by high doses of corticosteroids and possibly inflammation [8]. The most accurate tool for eGFR is proposed to be cysC [9], for risk stratification overall [7] and also in elderly individuals [9, 10], possibly because they typically have a low muscle mass.

Although other studies have investigated the association between kidney function and bone density (BMD), they are mostly in defined kidney disease [5]. In reviewing the literature, few studies include older women; most are cross-sectional, ethnically diverse and based on creatinine. Subsequently, there is a gap in knowledge regarding kidney function in the old and bone health. Furthermore, no longitudinal studies have evaluated whether reduced kidney function is associated with low BMD, bone markers and deranged mineral homeostasis in a normal population of older women followed over many years. Although poor kidney function is associated with mortality [6], for older women who survive into advanced age despite having poor kidney function, little is known about their bone health.

Given the hypothesized association between kidney function and skeletal health, this investigation in older women aims to be of relevance for clinicians with the following objectives: (1) to evaluate the association between kidney function and BMD; (2) to identify differences in BMD, bone loss and bone markers in women stratified by kidney function; and (3) to describe bone-related characteristics of elderly women surviving up to age 85 and whose kidney function remained stable during the 10-year follow-up. To understand the current knowledge base, we started with a non-systematic literature review. We then estimated kidney function using cysC with the CKD-EPI formula in 1044 Caucasian women from the population-based Osteoporosis Prospective Risk Assessment (OPRA) cohort. All women were 75 years at baseline with follow-up visits at ages 80 and 85 .

\section{Material and methods}

\section{Subjects}

The OPRA cohort is a single-center, longitudinal populationbased cohort of Caucasian women, randomly selected from city files of Malmö from 1995 to 1999 [11]. Aiming to capture the average community-dwelling older woman, no exclusion criteria were applied. A total of 1604 women received an invitation on their 75th birthday, and 1044 (65\%) attended at baseline with 715 and 382 returning for the 5- and 10-year follow-up, respectively. At age 80, reasons for non-attendance included the following: illness (31\%); sheltered living (5\%); no reason (53\%); other reasons (social reasons, walking problems or migration $(11 \%)$ ). At age 85 : illness $(56 \%)$; sheltered living (7\%); no reason (23\%); other reasons (14\%).

The analyses in this study are based only on data from women with eGFRcysC-values, corresponding to 981 at baseline, 685 at age 80 and 365 at age 85 . Reasons for missing analytical values include the following: lack of serum, could not provide a blood sample, hemolysis and failed analysis; reflecting a random loss across the cohort and not a systematic error or selection.

The study was approved by the Regional Ethical Review Board in Lund and performed in accordance with the Helsinki declaration. Participants provided written informed consent.

\section{Kidney function}

Plasma-cysC from all visits was analyzed in batch in 2015 at the Department of Clinical Chemistry, Malmö, Skåne University Hospital using Cobas auto-analyzer (CV 2.2$1.1 \%)$. Estimated glomerular function rate $\left(\mathrm{mL} / \mathrm{min} / 1.73 \mathrm{~m}^{2}\right)$ was calculated using the CKD-EPI cysC -formula [7]. Comparative analyses were also performed with creatininebased eGFR calculations using the CG- and MDRD-formulas, as previously described [6].

\section{BMD and bone loss}

Using dual-energy X-ray absorptiometry (DXA), measured with a Lunar DPX-L (GE Lunar, Madison, WI), areal BMD $\left(\mathrm{g} / \mathrm{cm}^{2}\right)$ was determined at femoral neck $(\mathrm{FN})$ and total body (TB) at Skåne University Hospital Malmö. Precision of DXA was assessed by duplicate measurements on healthy individuals. Precision error was $0.009-0.010 \mathrm{~g} / \mathrm{cm}^{2}$ at FN and $0.011-$ $0.030 \mathrm{~g} / \mathrm{cm}^{2}$ at TB. No drifts in phantom-measured results were observed [12]. Osteoporosis was defined as a Tscore $\leq-2.5$ at the FN and osteopenia as a T-score between -1 and -2.5 . Rate of bone loss was calculated individually for every woman as annual percentage change in BMD or as absolute bone loss over 5 years (between 75 and 80, $n=685$; between 80 and $85, n=365$ ) and 10 years (between 75 and 85 , $n=365$ ).

\section{Blood biochemistry}

Non-fasting blood samples were collected between 8 a.m. and 1 p.m., centrifuged and stored at $-80^{\circ} \mathrm{C}$. Plasma calcium and 
alkaline phosphate (ALP) were analyzed using a Beckman synchron LX20-4 auto-analyzer or Cobas auto-analyzer (Roche). At age 75, serum levels of PTH were analyzed using Elecsys PTH immunoassay (Roche Diagnostics, Mannheim, Germany). The normal range spanned from 1.6 to $6.9 \mathrm{pmol} / \mathrm{L}$ with intra- and inter-assay CVs of 1.6 and $5.7 \%$, respectively. At the 5- and 10-year follow-up, PTH was analyzed using Cobas auto-analyzer by Roche (intra-assay CV, 7-5\%). To ensure inter-assay comparability between methods, consistency of PTH values was assessed by duplicate measurements [13]. Plasma phosphate from all visits was analyzed in batch according to routine methods. Serum $25(\mathrm{OH}) \mathrm{D}$ (vitamin D) was analyzed using liquid chromatography mass spectrophotometry (LC-MS) (inter-assay CV 3-6\%). CRP was analyzed using Roche Diagnostics (Cobas) with a CV of 3.6-4.1\%. All assays were performed according to routine methods. Bone turnover markers S-TRACP5b, serum bone-specific alkaline phosphatase (s-BALP) and total osteocalcin were analyzed as previously described [14].

\section{Other variables}

Using standardized methods, weight $(\mathrm{kg})$ and height $(\mathrm{cm})$ were measured and BMI $\left(\mathrm{kg} / \mathrm{m}^{2}\right)$ calculated. Lifestylerelated information was available from questionnaires.

\section{Literature search}

For the purpose of comparison to other published studies, we made a non-systematic literature search in PubMed using the following: ((" "Renal Insufficiency"[All Fields] OR "Renal Insufficiency, Chronic" [Mesh] OR "estimated glomerular filtration rate"[All Fields]) AND "Bone Density"[All Fields]) AND ("female"[MeSH Terms] AND "aged"[MeSH Terms])) AND "longitudinal studies"[MeSH Terms]. This only resulted in 10 articles; therefore, the search term "longitudinal" was removed, resulting in 309 articles, of which 20 were deemed relevant.

\section{Statistics}

Analyses were performed using just the available data (Online resource 1a). Descriptive data is presented as mean with standard deviation or median with interquartile range as appropriate. To identify association between kidney function (eGFR) and BMD, multivariate linear regression analyses with eGFR (independent) and BMD (dependent) as continuous variables was performed, adjusted for weight (model 1) and weight, smoking and vitamin D (model 2). In each model, we present partial explained variation (unadjusted $R^{2}$ ) for eGFR. The analyses were also performed after excluding steroid ( $n=29$ at age $75 ; n=41$ at age $80 ; n=22$ at age 85) and bisphosphonate ( $n=33$ at age $75 ; n=58$ at age 80; $n=55$ at age 85 ) users. A previous report from the OPRA cohort indicated that having ever used a potent estrogen $(n=49,5 \%)$ has no effect on BMD at the age of 75 [15], and therefore, current users were not excluded from the analyses.

Participants were staged into categories of kidney function based on their eGFR alone as follows: (1) normalmild reduction of kidney function, eGFR $\geq 60 \mathrm{~mL} / \mathrm{min} /$ $1.73 \mathrm{~m}^{2}$ (CKD stages 1-2, "normal" kidney function), (2) mild-moderate reduction, eGFR from 45 to $59 \mathrm{~mL} / \mathrm{min} /$ $1.73 \mathrm{~m}^{2}$ (CKD stage 3A, "intermediate" function) and (3) moderate-severe reduction, eGFR $<45 \mathrm{~mL} / \mathrm{min} / 1.73 \mathrm{~m}^{2}$ (CKD stages 3B-5, "poor" function) [4].

Power analyses for bone density were performed prior to baseline inclusion; based on the supposition of $0.13 \mathrm{~g} /$ $\mathrm{cm}^{2} \mathrm{SD}$ in BMD, this study has over $80 \%$ power to detect a $0.056 \mathrm{~g} / \mathrm{cm}^{2}$ difference between equal groups at a $5 \%$ significance level. Differences in bone loss and characteristics according to kidney function were calculated using ANOVA (for normally distributed data) or Kruskal-Wallis (for non-normally distributed data). Differences between the women who maintained a normal versus poor kidney function throughout follow-up were compared using independent sample $t$ test or Mann-Whitney $U$-test.

All data was analyzed with SPSS (IBM SPSS v22.0; Armonk, NY: IBM Corp.). A $p$ value $<0.05$ was considered nominally significant. When difference between three groups is compared, significance is presented as $p$ values for trend.

\section{Results}

Characteristics of the OPRA population cross-sectionally and longitudinally for those continuing follow-up are shown in Table 1. Cross-sectionally, eGFR declined, as did FN and TB T-score at each assessment point and mirrored in those with who attended all visits. The number of women with osteoporosis increased from 28 to $49 \%$ during the follow-up. As described previously [6], women who attended follow-up tended to have higher baseline eGFR compared to non-attendees. Calcium and/or vitamin D supplement intake did not differ between categories of kidney function (Online resource 1b).

\section{Association between kidney function and bone mineral density}

Kidney function (eGFR by CKD-EPI $\mathrm{cysC}_{\text {C }}$ ) was positively associated with FN BMD at both 75 and 80 years old in the fully adjusted models $(\hat{\beta}=0.001, p=0.028$ and $\hat{\beta}=0.001$, 
Table 1 Clinical data at ages 75, 80 and 85 presented as cross-sectional and as longitudinal for those who successfully underwent long-term follow-up. Mean individual change (MIC) for the 5-year follow-ups is presented at ages 80 and 85

\begin{tabular}{|c|c|c|c|c|c|c|c|c|c|c|c|c|c|c|c|}
\hline & \multicolumn{6}{|c|}{ Cross-sectional } & \multicolumn{4}{|c|}{ Longitudinal at age $80(n=685)$} & \multicolumn{5}{|c|}{ Longitudinal at age $85(n=365)$} \\
\hline & \multicolumn{2}{|c|}{$\begin{array}{l}\text { Age } 75 \\
(n=981)\end{array}$} & \multicolumn{2}{|c|}{$\begin{array}{l}\text { Age } 80 \\
(n=685)\end{array}$} & \multicolumn{2}{|c|}{$\begin{array}{l}\text { Age } 85 \\
(n=365)\end{array}$} & \multirow{2}{*}{$\begin{array}{l}\text { Age } 75 \\
\text { Mean }\end{array}$} & \multicolumn{3}{|l|}{ Age 80} & \multirow{2}{*}{$\begin{array}{l}\text { Age } 75 \\
\text { Mean }\end{array}$} & \multirow{2}{*}{$\begin{array}{l}\text { Age } 80 \\
\text { Mean }\end{array}$} & \multicolumn{2}{|c|}{ Age 85} & \multirow[b]{2}{*}{$(\mathrm{SD})$} \\
\hline & Mean & $(\mathrm{SD})$ & Mean & $(\mathrm{SD})$ & Mean & (SD) & & Mean & MIC & (SD) & & & Mean & MIC & \\
\hline Age & 75.2 & $(0.1)$ & 80.2 & $(0.2)$ & 85.2 & $(0.14)$ & 75.2 & 80.2 & +5.0 & $(0.1)$ & 75.2 & 80.2 & 85.2 & +5.0 & $(0.1)$ \\
\hline Body mass index & 26.3 & $(4.2)$ & 26.0 & $(4.2)$ & 25.4 & $(4.0)$ & 26.3 & 26.0 & -0.3 & $(2.1)$ & 26.1 & 25.9 & 25.4 & -0.5 & $(1.8)$ \\
\hline Weight & 68 & (12) & 66 & (11) & 64 & (11) & 68 & 66 & -2 & (5) & 67 & 66 & 64 & -2 & (5) \\
\hline Height & 161 & $(6)$ & 159 & $(6)$ & 158 & (6) & 161 & 159 & -1 & (1) & 161 & 160 & 158 & -1 & (1) \\
\hline Femoral neck T-score & -1.8 & $(1.1)$ & -2.2 & $(1.1)$ & -2.4 & (1.1) & -1.7 & -2.2 & -0.5 & $(0.7)$ & -1.7 & -2.2 & -2.4 & -0.2 & $(0.8)$ \\
\hline Total body T-score & -1.6 & $(1.2)$ & -1.7 & $(1.2)$ & -1.7 & $(1.3)$ & -1.5 & -1.7 & -0.2 & $(0.4)$ & -1.5 & -1.7 & -1.7 & -0.1 & $(0.4)$ \\
\hline Osteopenia $(n)$ & 464 & $(49 \%)$ & 299 & $(44 \%)$ & 144 & $(39 \%)$ & 343 & 299 & -44 & & 174 & 160 & 144 & -16 & \\
\hline Osteoporosis $(n)$ & 269 & $(28 \%)$ & 300 & $(44 \%)$ & 181 & $(49 \%)$ & 180 & 300 & +120 & & 94 & 150 & 181 & +31 & \\
\hline \multicolumn{16}{|l|}{ Kidney function } \\
\hline p-CysC & 1.06 & $(0.31)$ & $1.18^{\mathrm{a}}$ & $(0.34)$ & $1.28^{\mathrm{a}}$ & $(0.39)$ & 1.08 & $1.18^{\mathrm{a}}$ & +0.15 & $(0.25)$ & 1.07 & $1.14^{\mathrm{a}}$ & $1.28^{\mathrm{a}}$ & +0.16 & $(0.24)$ \\
\hline $\mathrm{eGFR}^{\mathrm{b}}$ & 63 & (18) & 54 & $(15)$ & 47 & (14) & 65 & 54 & -11 & (12) & 66 & 56 & 47 & -9 & (10) \\
\hline Normal $(n)$ & 570 & $(58 \%)$ & 236 & $(34 \%)$ & 60 & $(16 \%)$ & 404 & 236 & -168 & & 226 & 128 & 60 & -68 & \\
\hline Intermediate $(n)$ & 252 & $(26 \%)$ & 265 & $(39 \%)$ & 148 & $(41 \%)$ & 173 & 265 & +92 & & 85 & 134 & 148 & +14 & \\
\hline Poor $(n)$ & 159 & $(16 \%)$ & 184 & $(27 \%)$ & 157 & $(43 \%)$ & 73 & 184 & +111 & & 35 & 74 & 157 & +83 & \\
\hline p-Calcium & 2.40 & $(0.07)$ & 2.41 & $(0.13)$ & 2.34 & $(0.09)$ & 2.41 & 2.41 & $-0.01^{\mathrm{a}}$ & $(0.1)$ & 2.40 & 2.40 & 2.34 & $-0.05^{\mathrm{a}}$ & $(0.1)$ \\
\hline p-Phosphate & 1.11 & $(0.23)$ & $1.09^{\mathrm{a}}$ & $(0.19)$ & $1.13^{\mathrm{a}}$ & $(0.17)$ & 1.12 & $1.09^{\mathrm{a}}$ & -0.02 & $(0.19)$ & 1.12 & 1.10 & $1.13^{\mathrm{a}}$ & 0.04 & $(0.15)$ \\
\hline s-PTH & 4.64 & $(2.05)$ & 5.04 & $(2.69)$ & $4.20^{\mathrm{a}}$ & (3.14) & 4.56 & 5.04 & $+0.6^{\mathrm{a}}$ & $(2.2)$ & $4.10^{\mathrm{a}}$ & 4.97 & $4.20^{\mathrm{a}}$ & $-0.5^{\mathrm{a}}$ & (2.4) \\
\hline $\mathrm{s}-25(\mathrm{OH}) \mathrm{D}$ & 62 & (19) & 78 & $(30)$ & 79 & (26) & 64 & 78 & +14 & $(25)$ & 64 & 79 & 79 & 0 & $(25)$ \\
\hline p-ALP & 1.3 & $(0.5)$ & $1.3^{\mathrm{a}}$ & $(0.4)$ & $1.1^{\mathrm{a}}$ & $(0.3)$ & 1.4 & $1.3^{\mathrm{a}}$ & -0.1 & $(0.3)$ & 1.4 & $1.3^{\mathrm{a}}$ & $1.1^{\mathrm{a}}$ & -0.1 & $(0.3)$ \\
\hline $\mathrm{p}$-CRP & $1.9^{\mathrm{a}}$ & (3.1) & $1.9^{\mathrm{a}}$ & (3.3) & $1.7^{\mathrm{a}}$ & $(2.7)$ & $1.7^{\mathrm{a}}$ & $1.9^{\mathrm{a}}$ & $0.0^{\mathrm{a}}$ & (1.7) & $1.8^{\mathrm{a}}$ & $2.0^{\mathrm{a}}$ & $1.7^{\mathrm{a}}$ & $-0.1^{\mathrm{a}}$ & (1.5) \\
\hline s-Osteocalcin & 8.1 & $(4.3)$ & 6.1 & $(4.0)$ & & NA & 7.7 & 6.1 & -1.6 & (3.3) & 8.5 & 6.0 & NA & & \\
\hline s-BALP & 21.0 & $(9.0)$ & $28.0^{\mathrm{a}}$ & $(13.0)$ & & NA & $21^{\mathrm{a}}$ & $28.0^{\mathrm{a}}$ & +7.4 & $(9.1)$ & $21^{\mathrm{a}}$ & $28^{\mathrm{a}}$ & NA & & \\
\hline s-TRAP5b & 3.4 & $(1.2)$ & 5.5 & $(2.0)$ & 3.8 & $(1.2)$ & 3.4 & 5.5 & +2.1 & (1.8) & 3.4 & 5.4 & 3.8 & -1.6 & $(2.0)$ \\
\hline Current smoker $(n)$ & 136 & $(14 \%)$ & 73 & $(11 \%)$ & 20 & $(6 \%)$ & 86 & 73 & -13 & & 27 & 23 & 20 & -3 & \\
\hline Steroid user $(n)$ & 29 & $(3 \%)$ & 33 & $(5 \%)$ & 17 & $(5 \%)$ & 29 & 33 & +4 & & 8 & 20 & 17 & -3 & \\
\hline $\begin{array}{l}\text { Bisphosphonate user } \\
\quad(n)\end{array}$ & 32 & $(3 \%)$ & 47 & $(7 \%)$ & 44 & $(12 \%)$ & 24 & 47 & +23 & & 12 & 29 & 44 & +15 & \\
\hline
\end{tabular}

Age (years), body mass index $\left(\mathrm{kg} / \mathrm{m}^{2}\right)$, weight $(\mathrm{kg})$, height $(\mathrm{cm}), \mathrm{p}$-cysC $(\mathrm{mg} / \mathrm{L})$, eGFR $* *\left(\mathrm{~mL} / \mathrm{min} / 1.73 \mathrm{~m}{ }^{2}\right)$, p-calcium (mmol/L), p-phosphate (mmol/L), s-PTH (pmol/L), s-25(OH)D (nmol/L), p-ALP (ukat/L), p-CRP (mg/L), s-osteocalcin ( $\mu \mathrm{g} / \mathrm{L})$, s-BALP (U/L), s-TRAP5b (U/L)

${ }^{a}$ Median with IQR

${ }^{\mathrm{b}}$ eGFR estimated using CKD-EPI ${ }_{\text {cysC }}$

$p=0.001$, respectively), but not in advanced old age (85 years). Overall, the contribution was small; however, the partial explained variation, $0.4 \%$ at 75 years old and $1.6 \%$ at age 80 years.

Excluding women on bisphosphonates did not substantially alter the results, but with exclusion of steroid users, the association at age 75 was lost. Notably, kidney function and TB BMD were inversely associated at all three ages, although abolished with weight adjustment (Table 2). Comparative analyses using the creatinine-based formulas were essentially the same (Online resource 2).

\section{Bone loss and markers of bone metabolism stratified by kidney function}

To compare differences in bone loss and markers of bone metabolism, women were stratified into three categories (normal, intermediate, poor) based on their kidney function. To evaluate age-related associations, bone loss was compared during two different 5-year periods (between ages $75-80$ and $80-85$ ). Individually calculated annual percentage bone loss differed between the three categories ((FN $p=0.001$, Fig. 1); TB $p=0.014)$; a higher rate was 
Table 2 Association between kidney function (eGFR) and BMD at femoral neck and total body at age 75,80 and 85

\begin{tabular}{|c|c|c|c|}
\hline \multirow[t]{2}{*}{ BMD-FN } & \multicolumn{2}{|l|}{ CKD-EPI ${ }_{\text {cysC }}$} & \multirow[b]{2}{*}{$\begin{array}{l}\text { Partial explainec } \\
\text { variation }\end{array}$} \\
\hline & $\hat{\beta}(95 \% \mathrm{CI})$ & $p$-value & \\
\hline \multicolumn{4}{|c|}{ Age $75(n=981)$} \\
\hline Unadjusted & $-0.2 \times 10^{-3}\left(-0.7 \times 10^{-3}\right.$ to $\left.0.3 \times 10^{-3}\right)$ & 0.478 & $0.1 \%$ \\
\hline Model 1 & $0.4 \times 10^{-3}\left(-0.01 \times 10^{-3}\right.$ to $\left.0.9 \times 10^{-3}\right)$ & $0.056^{\S}$ & $0.3 \%$ \\
\hline Model 2 & $0.5 \times 10^{-3}\left(0.06 \times 10^{-3}\right.$ to $\left.1.0 \times 10^{-3}\right)$ & $0.028^{000} \S$ & $0.4 \%$ \\
\hline \multicolumn{4}{|c|}{ Age $80(n=685)$} \\
\hline Unadjusted & $0.3 \times 10^{-3}\left(-0.3 \times 10^{-3}\right.$ to $\left.1.0 \times 10^{-3}\right)$ & 0.323 & $0.1 \%$ \\
\hline Model 1 & $1.1 \times 10^{-3}\left(0.5 \times 10^{-3}\right.$ to $\left.1.7 \times 10^{-3}\right)$ & $<0.001^{\alpha \S}$ & $1.7 \%$ \\
\hline Model 2 & $1.1 \times 10^{-3}\left(0.5 \times 10^{-3}\right.$ to $\left.1.8 \times 10^{-3}\right)$ & $0.001^{\alpha \propto}$ & $1.6 \%$ \\
\hline \multicolumn{4}{|c|}{ Age $85(n=366)$} \\
\hline Unadjusted & $-0.2 \times 10^{-3}\left(-1.2 \times 10^{-3}\right.$ to $\left.0.9 \times 10^{-3}\right)$ & 0.778 & $0.0 \%$ \\
\hline Model 1 & $0.6 \times 10^{-3}\left(-0.4 \times 10^{-3}\right.$ to $\left.1.6 \times 10^{-3}\right)$ & 0.219 & $0.4 \%$ \\
\hline Model 2 & $0.6 \times 10^{-3}\left(-0.4 \times 10^{-3}\right.$ to $\left.1.7 \times 10^{-3}\right)$ & 0.238 & $0.3 \%$ \\
\hline \multirow[t]{2}{*}{ BMD-TB } & \multicolumn{2}{|l|}{ CKD-EPI ${ }_{\text {cysC }}$} & \multirow[b]{2}{*}{$\begin{array}{l}\text { Partial explained } \\
\text { variation }\end{array}$} \\
\hline & $\hat{\beta}(95 \% \mathrm{CI})$ & p-value & \\
\hline \multicolumn{4}{|c|}{ Age $75(n=981)$} \\
\hline Unadjusted & $-0.4 \times 10^{-3}\left(-0.8 \times 10^{-3}\right.$ to $\left.-0.3 \times 10^{-4}\right)$ & $0.034^{a} \S \S$ & $0.5 \%$ \\
\hline Model 1 & $0.03 \times 10^{-4}\left(-0.3 \times 10^{-3}\right.$ to $\left.0.3 \times 10^{-3}\right)$ & 0.830 & $0.0 \%$ \\
\hline Model 2 & $0.04 \times 10^{-4}\left(-0.3 \times 10^{-3}\right.$ to $\left.0.3 \times 10^{-3}\right)$ & 0.796 & $0.0 \%$ \\
\hline \multicolumn{4}{|c|}{ Age $80(n=685)$} \\
\hline Unadjusted & $-0.6 \times 10^{-3}\left(-1.1 \times 10^{-3}\right.$ to $\left.-0.1 \times 10^{-3}\right)$ & $0.019^{\mathrm{a}} \S$ & $0.8 \%$ \\
\hline Model 1 & $0.1 \times 10^{-3}\left(-0.3 \times 10^{-3}\right.$ to $\left.0.6 \times 10^{-3}\right)$ & 0.559 & $0.1 \%$ \\
\hline Model 2 & $0.1 \times 10^{-3}\left(-0.3 \times 10^{-3}\right.$ to $\left.0.6 \times 10^{-3}\right)$ & 0.610 & $0.0 \%$ \\
\hline \multicolumn{4}{|c|}{ Age $85(n=366)$} \\
\hline Unadjusted & $-1.0 \times 10^{-3}\left(-1.8 \times 10^{-3}\right.$ to $\left.-0.2 \times 10^{-3}\right)$ & $0.012^{\mathrm{\alpha} \S}$ & $1.7 \%$ \\
\hline Model 1 & $-0.2 \times 10^{-3}\left(-0.8 \times 10^{-3}\right.$ to $\left.0.5 \times 10^{-3}\right)$ & 0.609 & $0.1 \%$ \\
\hline Model 2 & $-0.3 \times 10^{-3}\left(-1.0 \times 10^{-3}\right.$ to $\left.0.4 \times 10^{-3}\right)$ & 0.410 & $0.2 \%$ \\
\hline
\end{tabular}

Model 1 adjusted for: weight

Model 2 adjusted for: weight, smoking, Vitamin-D

$P$-values calculated using linear regression analyses

§Significant when bisphosphonate users excluded ( $n=32$ at age $75, n=47$ at age $80, n=44$ at age 85 )

$\S \S$ Non-significant when bisphosphonate users excluded

a Significant when steroid users excluded ( $n=29$ at age $75, n=33$ at age 80, $n=17$ at age 85)

Non-significant when steroid users excluded apparent in women with intermediate (stage 3A) and poor (stages 3B-5) kidney function in the first time period, at both measurement sites. In the second period, bone loss did not differ at the FN $(p=0.264)$. However, a difference between the three categories was observed at TB $(p=0.044)$, and the lowest bone loss was seen in women with intermediate kidney function. During the first 5-year time period, analyzing absolute bone loss $\left(\mathrm{g} / \mathrm{cm}^{2} / 5\right.$ years $)$, the results were similar (data not shown).
Excluding bisphosphonate or steroid users at baseline did not significantly change the bone loss results between ages 75 and 80 . However, at age 80 , with exclusion of current bisphosphonate users, FN bone loss (between 80 and 85), differed between the groups ( $p$ for trend 0.048).

Table 3 shows characteristics of women stratified by kidney function at each age $(75,80$ and 85$)$. Although women with reduced kidney function (i.e. intermediate and poor) tended to have higher TB BMD $(p<0.05)$, there was no 
a

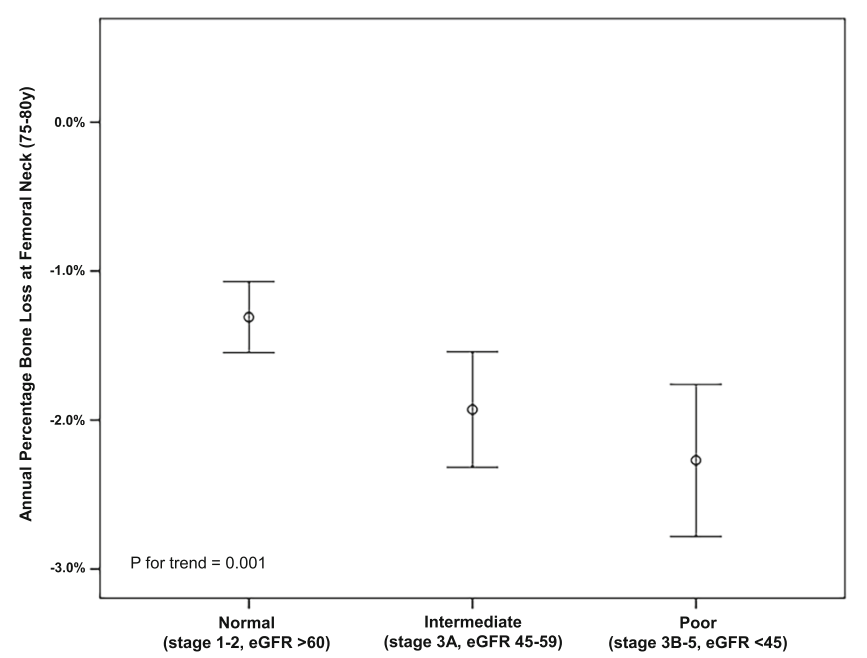

b

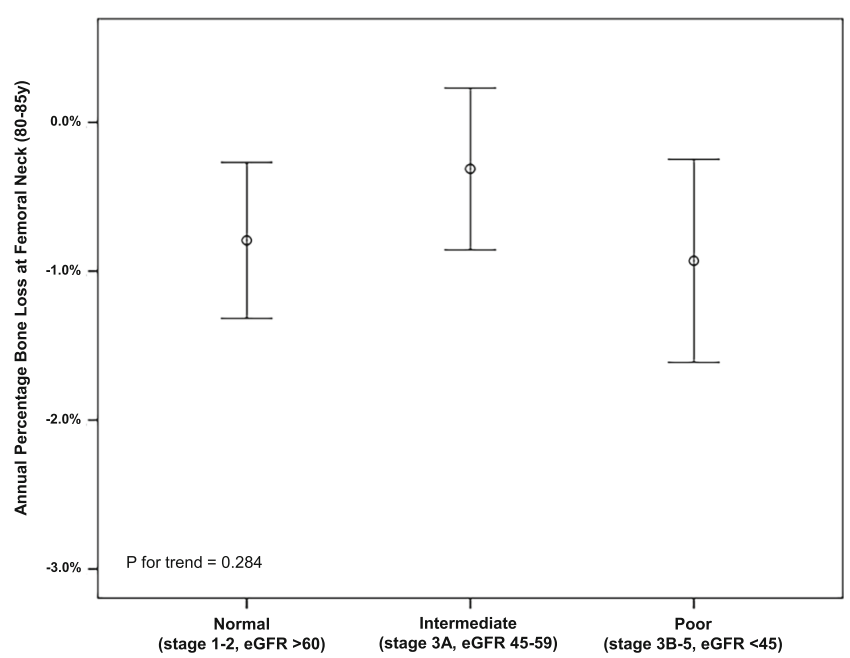

Fig. 1 Annual percentage bone loss over 5 years at the femoral neck. (a) Kidney function at 75 years and bone loss between 75 and 80 . (b) Kidney function at 80 years and bone loss

difference in FN BMD or prevalence of osteopenia and osteoporosis at any age.

Markers of mineral homeostasis also differed by kidney function; at all ages, PTH was higher as kidney function decreased $(p<0.001)$, although mean values were well within normal range. However, phosphate and ALP only differed at age $75(p<0.001)$, although women with poor function developed higher calcium levels. Furthermore, at all ages, women with reduced kidney function had higher values for weight, the inflammation marker CRP and the bone formation marker osteocalcin $(p<0.001$ for all). Other bone markers, BALP and TRACP5, did not differ with kidney function.

\section{Characteristics of women whose CKD stage remained stable over 10 years}

To understand the skeletal implications of kidney function over time, we compared women who had, and maintained, poor kidney function (stages $3 \mathrm{~b}-5, n=26$ ) throughout the 10 -year observation period to those with normal function throughout (stages 1-2, $n=52$ ). Women who survived despite poor function had higher annual percentage bone loss at the FN over the 10 years, although variability precluded statistical significance ( $p=0.097$ ) (Fig. 2). Using absolute bone loss values, the results were largely similar.

Continuous poor kidney function was associated with a higher TB BMD ( $p<0.05$ all time points). FN BMD was higher, and correspondingly, prevalence of osteoporosis lower, although only significant at baseline ( $p=0.039$ and $p=0.017$, respectively; Table 4). In addition, the women with continuous poor function had higher PTH (increasing even above normal range at age 85) and were $10 \mathrm{~kg}$ heavier
( $p<0.05$ and $p<0.001$, respectively, for all time points). Differences in calcium, phosphate, CRP, BALP and osteocalcin were also observed.

\section{Review of the literature}

To put our results into context and demonstrate the current knowledge base for kidney function and BMD or bone loss in elderly women, the findings from the literature review are summarized in Online resource 3 (in all 20 studies). A coherent picture of kidney function and $\mathrm{BMD} /$ mineral homeostasis is hampered by heterogeneity in study populations (wide age spans, ethnicity and number of participants). Only ten studies comprised more than 200 participants, and only four represent Caucasian women. Seven studies have been performed in patients with defined $\mathrm{CKD}$, with a mean age considerably below 75 years. Furthermore, only three studies were longitudinal, and none of these had $>5$ years of follow-up. Most studies used the creatinine-based MDRD or CG-formulas to estimate GFR, making comparisons difficult [16-35].

\section{Discussion}

Kidney function decreases with age [36], and although chronic kidney disease is associated with deranged mineral homeostasis [5] potentially affecting bone mass, this is to our knowledge the first study extending over a decade focusing on very elderly women, a group already at high risk of osteoporosis. In this study, we followed kidney function and bone loss for 10 years in the large population-based cohort and show that declining kidney function is adversely associated with bone 
Table 3 OPRA clinical data categorized by kidney function ${ }^{\mathrm{a}}$ at ages 75,80 and 85

\begin{tabular}{|c|c|c|c|c|}
\hline & $\begin{array}{l}\text { Normal } \\
\text { Mean }(95 \% \mathrm{CI})\end{array}$ & $\begin{array}{l}\text { Intermediate } \\
\text { Mean }(95 \% \mathrm{CI})\end{array}$ & $\begin{array}{l}\text { Poor } \\
\text { Mean }(95 \% \mathrm{CI})\end{array}$ & $p$ for trend \\
\hline Age 75 & $n=570$ & $n=252$ & $n=159$ & \\
\hline eGFR $\left(\mathrm{mL} / \mathrm{min} / 1.73 \mathrm{~m}^{2}\right)$ & $75(74-76)$ & $53(53-54)$ & $36(35-37)$ & \\
\hline Weight $(\mathrm{kg})$ & $66(65-67)$ & $70(69-72)$ & $69(68-71)$ & $<0.001$ \\
\hline TB-BMD $\left(\mathrm{g} / \mathrm{cm}^{2}\right)$ & $1.000(0.992-1.009)$ & $1.020(1.006-1.033)$ & $1.012(0.995-1.028)$ & 0.039 \\
\hline FN-BMD $\left(\mathrm{g} / \mathrm{cm}^{2}\right)$ & $0.762(0.750-0.773)$ & $0.775(0.757-0.793)$ & $0.761(0.739-0.784)$ & 0.441 \\
\hline Osteopenia (n) & $261(48 \%)$ & $114(50 \%)$ & $62(48 \%)$ & 0.915 \\
\hline Osteoporosis (n) & $161(30 \%)$ & $57(25 \%)$ & $37(29 \%)$ & 0.422 \\
\hline $\mathrm{p}-\mathrm{Ca}(\mathrm{mmol} / \mathrm{L})$ & $2.40(2.40-2.41)$ & $2.40(2.40-2.41)$ & $2.42(2.40-2.43)$ & 0.211 \\
\hline p-Phosphate $(\mathrm{mmol} / \mathrm{L})$ & $1.09(1.08-1.10)$ & $1.12(1.10-1.14)$ & $1.27(1.23-1.30)$ & $<0.001$ \\
\hline $\mathrm{s}-\mathrm{PTH}(\mathrm{pmol} / \mathrm{L})$ & $4.4(4.2-4.5)$ & $4.6(4.3-4.9)$ & $5.7(5.2-6.1)$ & $<0.001$ \\
\hline $\mathrm{s}-25(\mathrm{OH}) \mathrm{D}(\mathrm{nmol} / \mathrm{L})$ & $62(60-63)$ & $63(60-65)$ & $62(58-65)$ & 0.752 \\
\hline $\mathrm{p}-\mathrm{ALP}^{\mathrm{b}}(\mathrm{ukat} / \mathrm{L})$ & $1.31(0.46)$ & $1.40(0.44)$ & $1.45(0.53)$ & $<0.001$ \\
\hline $\mathrm{p}-\mathrm{CRP}^{\mathrm{b}}(\mathrm{mg} / \mathrm{L})$ & $1.5(2.6)$ & $2.2(3.1)$ & $3.3(5.5)$ & $<0.001$ \\
\hline $\mathrm{s}$ Osteocalcin ${ }^{\mathrm{b}}(\mu \mathrm{g} / \mathrm{L})$ & $7.6(4.0)$ & $8.2(4.3)$ & $9.6(6.0)$ & $<0.001$ \\
\hline $\mathrm{s}-\mathrm{BALP}^{\mathrm{b}}(\mathrm{U} / \mathrm{L})$ & $21.0(9.0)$ & $21.0(9.0)$ & $22.0(10.0)$ & 0.224 \\
\hline s-TRACP5b (U/L) & $3.4(3.3-3.5)$ & $3.3(3.2-3.5)$ & $3.5(3.3-3.7)$ & 0.333 \\
\hline Age 80 & $n=236$ & $n=265$ & $n=184$ & \\
\hline $\mathrm{eGFR}\left(\mathrm{mL} / \mathrm{min} / 1.73 \mathrm{~m}^{2}\right)$ & $71(70-72)$ & $52(52-53)$ & $37(36-38)$ & \\
\hline Weight $(\mathrm{kg})$ & $63(62-64)$ & $67(66-68)$ & $69(67-71)$ & $<0.001$ \\
\hline TB-BMD $\left(\mathrm{g} / \mathrm{cm}^{2}\right)$ & $0.984(0.971-0.997)$ & $1.003(0.991-1.015)$ & $1.007(0.992-1.021)$ & 0.038 \\
\hline FN-BMD $\left(\mathrm{g} / \mathrm{cm}^{2}\right)$ & $0.713(0.696-0.730)$ & $0.723(0.708-0.739)$ & $0.705(0.685-0.725)$ & 0.328 \\
\hline Osteopenia $(n / \%)$ & $93(41 \%)$ & $125(49 \%)$ & $73(42 \%)$ & 0.752 \\
\hline Osteoporosis $(n / \%)$ & $105(47 \%)$ & $98(39 \%)$ & $79(45 \%)$ & 0.836 \\
\hline $\mathrm{p}-\mathrm{Ca}(\mathrm{mmol} / \mathrm{L})$ & $2.41(2.39-2.42)$ & $2.40(2.38-2.41)$ & $2.44(2.42-2.46)$ & 0.004 \\
\hline p-Phosphate $(\mathrm{mmol} / \mathrm{L})$ & $1.11(1.09-1.13)$ & $1.08(1.07-1.10)$ & $1.09(1.07-1.12)$ & 0.157 \\
\hline $\mathrm{s}-\mathrm{PTH}^{\mathrm{b}}(\mathrm{pmol} / \mathrm{L})$ & $4.2(2.2)$ & $4.9(2.9)$ & $5.1(3.7)$ & $<0.001$ \\
\hline $\mathrm{s}-25(\mathrm{OH}) \mathrm{D}(\mathrm{nmol} / \mathrm{L})$ & $76(72-80)$ & $76(73-80)$ & $82(77-87)$ & $<0.001$ \\
\hline p-ALP ${ }^{\mathrm{b}}($ ukat $/ \mathrm{L})$ & $1.23(0.41)$ & $1.26(0.46)$ & $1.28(0.45)$ & 0.197 \\
\hline $\mathrm{p}-\mathrm{CRP}^{\mathrm{b}}(\mathrm{mg} / \mathrm{L})$ & $1.4(2.7)$ & $2.0(3.2)$ & $2.8(4.4)$ & $<0.001$ \\
\hline s-Osteocalcin $(\mu \mathrm{g} / \mathrm{L})$ & $6.1(5.7-6.4)$ & $6.9(6.5-7.3)$ & $8.1(7.4-8.9)$ & $<0.001$ \\
\hline $\mathrm{s}-\mathrm{BALP}^{\mathrm{b}}(\mathrm{U} / \mathrm{L})$ & $28.0(12.0)$ & $28.0(14.0)$ & $27.0(11.8)$ & 0.215 \\
\hline s-TRACP5b (U/L) & $5.7(5.4-5.9)$ & $5.5(5.2-5.7)$ & $5.4(5.1-5.7)$ & 0.405 \\
\hline Age 85 & $n=60$ & $n=148$ & $n=157$ & \\
\hline $\mathrm{eGFR}\left(\mathrm{mL} / \mathrm{min} / 1.73 \mathrm{~m}^{2}\right)$ & $68(67-70)$ & $52(51-53)$ & $35(34-36)$ & \\
\hline Weight $(\mathrm{kg})$ & $60(57-62)$ & $63(62-65)$ & $66(64-68)$ & $<0.001$ \\
\hline TB-BMD (g/cm²) & $0.966(0.941-0.991)$ & $0.986(0.971-1.000)$ & $1.007(0.989-1.024)$ & 0.024 \\
\hline FN-BMD $\left(\mathrm{g} / \mathrm{cm}^{2}\right)$ & $0.682(0.648-0.718)$ & $0.684(0.664-0.705)$ & $0.696(0.672-0.720)$ & 0.709 \\
\hline Osteopenia $(n / \%)$ & $23(40 \%)$ & $56(39 \%)$ & $57(38 \%)$ & 0.264 \\
\hline Osteoporosis $(n / \%)$ & $29(50 \%)$ & $75(52 \%)$ & $71(47 \%)$ & 0.179 \\
\hline $\mathrm{p}-\mathrm{Ca}(\mathrm{mmol} / \mathrm{L})$ & $2.31(2.29-2.32)$ & $2.34(2.32-2.36)$ & $2.36(2.34-2.37)$ & 0.002 \\
\hline p-Phosphate ${ }^{\mathrm{b}}(\mathrm{mmol} / \mathrm{L})$ & $1.14(0.16)$ & $1.13(0.15)$ & $1.14(0.17)$ & 0.901 \\
\hline $\mathrm{s}-\mathrm{PTH}^{\mathrm{b}}(\mathrm{pmol} / \mathrm{L})$ & $3.2(2.1)$ & $4.1(2.9)$ & $4.7(4.3)$ & $<0.001$ \\
\hline $\mathrm{s}-25(\mathrm{OH}) \mathrm{D}(\mathrm{nmol} / \mathrm{L})$ & $81(73-88)$ & $76(73-80)$ & $80(75-85)$ & 0.389 \\
\hline $\mathrm{p}-\mathrm{ALP}^{\mathrm{b}}(\mathrm{ukat} / \mathrm{L})$ & $1.20(0.30)$ & $1.10(0.40)$ & $1.20(0.40)$ & 0.077 \\
\hline $\mathrm{p}-\mathrm{CRP}^{\mathrm{b}}(\mathrm{mg} / \mathrm{L})$ & $1.1(2.3)$ & $1.5(2.3)$ & $2.1(3.7)$ & 0.001 \\
\hline s-Osteocalcin $(\mu \mathrm{g} / \mathrm{L})$ & NA & NA & NA & \\
\hline s-BALP (U/L) & NA & NA & NA & \\
\hline s-TRACP5b (U/L) & $3.9(3.5-4.2)$ & $3.9(3.7-4.1)$ & $3.8(3.6-4.0)$ & 0.784 \\
\hline
\end{tabular}

$p$ values calculated using ANOVA, Kruskal-Wallis or Chi-2 test

Osteoporosis is defined as a FN T-score $\leq-2.5$. Osteopenia is defined as a FN T-score $>-2.5$ and $<-1.0$

${ }^{\text {a }}$ Normal kidney function, stages $1-2$, eGFR $\geq 60 \mathrm{~mL} / \mathrm{min} / 1.73 \mathrm{~m}^{2}$; intermediate kidney function, stage $3 \mathrm{~A}$, eGFR $45-59 \mathrm{~mL} / \mathrm{min} / 1.73 \mathrm{~m}{ }^{2}$; poor kidney function, stages 3B-5 eGFR $<45 \mathrm{~mL} / \mathrm{min} / 1.73 \mathrm{~m}^{2}$

${ }^{\mathrm{b}}$ Median with interquartile range

mass in older women, although the association appears to attenuate with advanced aging.

In trying to elucidate the implications of kidney function on BMD in elderly women, we began by performing a nonsystematic literature search. Although lack of longitudinal studies and differences in study populations make comparison of results difficult, this search indicated an association between kidney function and BMD [17-21, 23, 28, 30], though whether mediated through differences in sex, age and weight is unclear $[18,19]$. The homogeneity of the 
Fig. 2 Annual percentage bone loss over 10 years $(75-85)$ at the femoral neck in women maintaining NORMAL or POOR kidney function* during the 10 years of follow-up

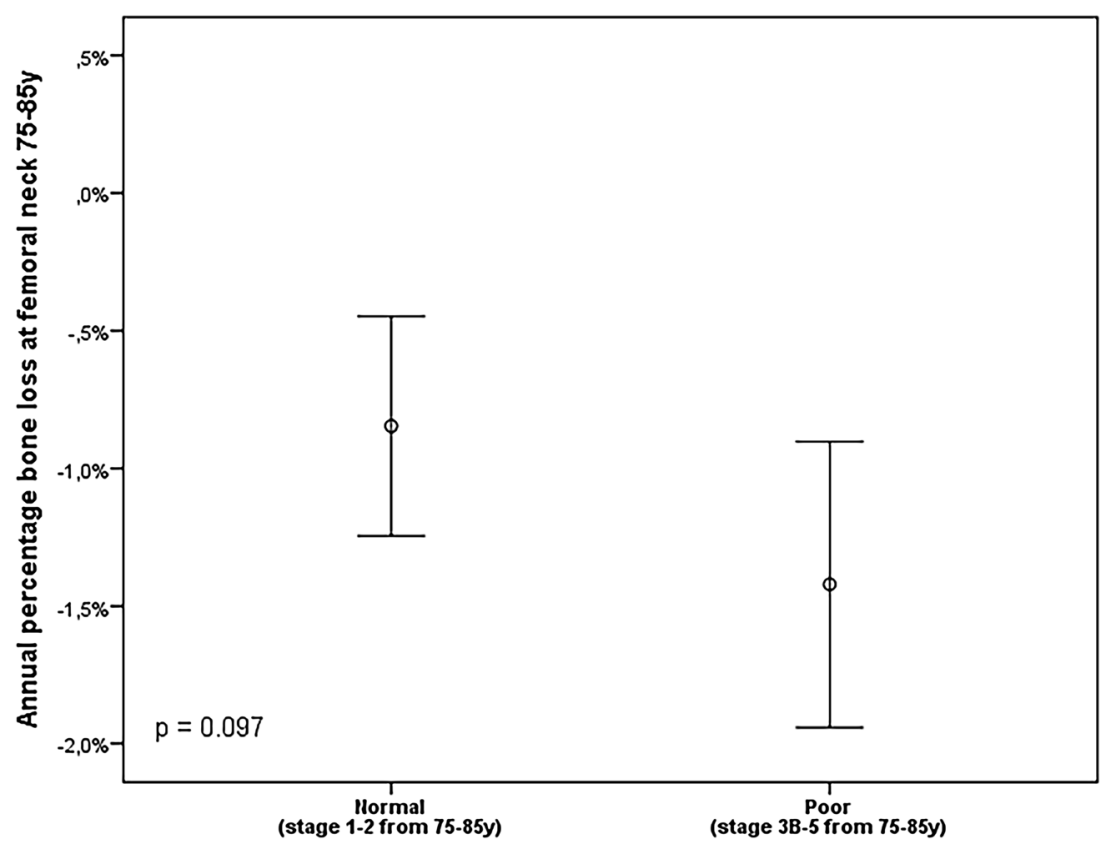

OPRA cohort, followed for 10 years, allowed us to address this gap in knowledge and make reliable conclusions about the association between kidney function and BMD in this elderly age group.

In this cohort, kidney function explains only a small proportion of the variation in FN BMD. Since others report stronger associations [20, 21, 23], we initially assumed the discrepancy could be explained by the use of creatinine-based eGFR formulas; yet, our results using either creatinine or cysC were largely similar. It is also possible that the contribution from kidney function to BMD is manifested at a much earlier age, or that in women aged 75-85, BMD is already so low that a further reduction regardless of cause will be small. The lack of association between kidney function and BMD in advanced age ( 85 years) or bone loss suggests that the association between kidney function and bone diminishes with age, reflecting an influence of health bias or a reduction in power with follow-up.

In postmenopausal women, higher hip and spine BMD [37] and reduced risk of hip fractures are commonly associated with higher BMI [38]. Accordingly, and highlighting the importance of weight for older women, kidney function was inversely associated with TB BMD before adjustments for weight. Indeed, reduced kidney function was associated with higher weight, perhaps explaining why these women tended to have a lower prevalence of osteoporosis.

To further understand the clinical implications of kidney function on bone loss in older women, we used the CKD staging of kidney function. In agreement with the Rancho Bernado Study [20], reduced kidney function (stages $3 a$ and 3b-5) was associated with higher hip bone loss, though not in advanced age ( $80-85$ years). The clinical relevance of this is uncertain and might be small in comparison with the contribution from other risk factors in this age group, since cross-sectionally, FN BMD did not differ between CKD stages. Why rate of bone loss was higher in the first (75-80 years) compared to the second ( $80-85$ years) time period is unclear, but we assume that bone loss will not be linear over long time spans, with a steady state reached between periods of loss. The lack of association between kidney function and BMD at age 85 and subsequent bone loss indicates that at a certain age, identifying risk factors with pronounced contribution becomes more difficult.

In the OPRA cohort, PTH and osteocalcin were incrementally higher the lower the kidney function - of clinical relevance not just for bone health, since increased PTH has been associated with cardiovascular disease [39] and mortality independent of kidney function [40]. However, mean PTH was well within reference range at all time points, and although we hypothesized that even slightly higher levels of PTH would be associated with higher bone resorption markers, it is not certain that this is the case in early CKD, and it seems not to be the case in the OPRA. In addition, and demonstrating that even a moderate reduction in kidney function affects bone metabolism in elderly women, reduced kidney function was associated with increased markers of bone metabolism. Lower calcium levels are associated with reduced kidney function, although it is unlikely that levels will be affected until eGFR falls below $30 \mathrm{~mL} / \mathrm{min} / 1.73 \mathrm{~m}^{2}$ (stages 4-5) [41] and very few women in our cohort reached that level of kidney disease. Opinions diverge regarding cysC and inflammation [8]; the women with reduced kidney function (stages $3 \mathrm{a}$ and $3 \mathrm{~b}-5$ ) had higher levels of CRP in this cohort.

We were interested to see what characterized women whose kidney function (either poor or normal) remained 
Table 4 Clinical characteristics of the women maintaining NORMAL or POOR kidney function ${ }^{\text {a }}$ during the 10 years of follow-up

\begin{tabular}{|c|c|c|c|c|c|c|c|c|c|}
\hline & \multicolumn{2}{|l|}{ Age 75} & \multirow[b]{2}{*}{$p$} & \multicolumn{2}{|l|}{ Age 80} & \multirow[b]{2}{*}{$p$} & \multicolumn{2}{|l|}{ Age 85} & \multirow[b]{2}{*}{$p$} \\
\hline & $\begin{array}{l}\text { Normal } \\
\text { function (eGFR } \\
\geq 60 \text { ) } \\
\text { Mean (SD) }\end{array}$ & $\begin{array}{l}\text { Poor function } \\
(\text { eGFR < 45) } \\
\text { Mean (SD) }\end{array}$ & & $\begin{array}{l}\text { Normal } \\
\text { function (eGFR } \\
\geq 60 \text { ) } \\
\text { Mean (SD) }\end{array}$ & $\begin{array}{l}\text { Poor function } \\
(\text { eGFR < 45) } \\
\text { Mean (SD) }\end{array}$ & & $\begin{array}{l}\text { Normal } \\
\text { function (eGFR } \\
\geq 60 \text { ) } \\
\text { Mean (SD) }\end{array}$ & $\begin{array}{l}\text { Poor function } \\
(\text { eGFR < 45) } \\
\text { Mean (SD) }\end{array}$ & \\
\hline $\begin{array}{l}\text { eGFR } \\
(\mathrm{mL} / \mathrm{min} / 1.73- \\
\left.\mathrm{m}^{2}\right)\end{array}$ & $83(11)$ & $37(6)$ & & $74(9)$ & $40(12)$ & & $69(7)$ & $33(8)$ & \\
\hline Weight $(\mathrm{kg})$ & $63(10)$ & $73(8)$ & $<0.001$ & $62(10)$ & $73(11)$ & $<0.001$ & $60(10)$ & $70(11)$ & $<0.001$ \\
\hline TB-BMD $\left(\mathrm{g} / \mathrm{cm}^{2}\right)$ & $0.991(0.092)$ & $1.063(0.078)$ & 0.001 & $0.981(0.098)$ & $1.038(0.080)$ & 0.021 & $0.965(0.094)$ & $1.054(0.083)$ & $<0.001$ \\
\hline FN-BMD $\left(\mathrm{g} / \mathrm{cm}^{2}\right)$ & $0.759(0.138)$ & $0.828(0.118)$ & 0.039 & $0.717(0.113)$ & $0.762(0.124)$ & 0.131 & $0.688(0.133)$ & $0.724(0.125)$ & 0.270 \\
\hline Osteopenia $(n)$ & $23(45 \%)$ & $13(54 \%)$ & 0.514 & $22(46 \%)$ & $12(50 \%)$ & 0.546 & $19(37 \%)$ & $10(42 \%)$ & 0.522 \\
\hline Osteoporosis $(n)$ & $17(33 \%)$ & $2(8 \%)$ & 0.017 & $20(42 \%)$ & $7(29 \%)$ & 0.240 & $26(51 \%)$ & $9(38 \%)$ & 0.215 \\
\hline $\mathrm{p}-\mathrm{Ca}(\mathrm{mmol} / \mathrm{L})$ & $2.38(0.06)$ & $2.42(0.07)$ & 0.008 & $2.36^{\mathrm{b}}(0.11)$ & $2.41^{\mathrm{b}}(0.09)$ & 0.091 & $2.30(0.06)$ & $2.37(0.11)$ & 0.005 \\
\hline $\begin{array}{r}\text { p-Phosphate } \\
(\mathrm{mmol} / \mathrm{L})\end{array}$ & $1.13(0.16)$ & $1.27(0.22)$ & 0.002 & $1.12(0.13)$ & $1.07(0.13)$ & 0.076 & $1.11(0.13)$ & $1.10(0.10)$ & 0.661 \\
\hline s-PTH (pmol/L) & $3.7^{\mathrm{b}}(2.3)$ & $4.9^{\mathrm{b}}(3.0)$ & 0.006 & $4.3(1.8)$ & $6.1(3.8)$ & 0.038 & $3.5(1.6)$ & $7.2(5.8)$ & 0.006 \\
\hline $\begin{array}{l}\mathrm{S}-25(\mathrm{OH}) \mathrm{D} \\
(\mathrm{nmol} / \mathrm{L})\end{array}$ & $63(20)$ & $66(17)$ & 0.402 & $79(27)$ & $74(29)$ & 0.446 & $79(29)$ & $84(33)$ & 0.518 \\
\hline p-ALP (ukat/L) & $1.3(0.4)$ & $1.5(0.5)$ & 0.069 & $1.3(0.3)$ & $1.4(0.6)$ & 0.379 & $1.2(0.3)$ & $1.2(0.3)$ & 0.834 \\
\hline p-CRP (mg/L) & $1.2^{\mathrm{b}}(2.0)$ & $2.5^{\mathrm{b}}(2.7)$ & 0.007 & $1.2^{\mathrm{b}}(2.0)$ & $3.3^{\mathrm{b}}(5.6)$ & 0.013 & $1.1^{\mathrm{b}}(2.6)$ & $3.0^{\mathrm{b}}(3.6)$ & 0.131 \\
\hline $\begin{array}{l}\text { s-Osteocalcin } \\
\quad(\mu \mathrm{g} / \mathrm{L})\end{array}$ & $7.5(2.8)$ & $10.1(4.0)$ & 0.001 & $6.0(2.5)$ & $8.7(5.3)$ & 0.026 & NA & NA & \\
\hline s-BALP (U/L) & $20.7(6.5)$ & $26.0(11.8)$ & 0.040 & $28.0^{\mathrm{b}}(15.5)$ & $29.0^{\mathrm{b}}(15.8)$ & 0.558 & NA & NA & \\
\hline s-TRACP5b (U/L) & $3.3(1.1)$ & $3.6(1.0)$ & 0.280 & $5.5(1.7)$ & $5.1(2.4)$ & 0.457 & $3.9(1.3)$ & $3.7(1.1)$ & 0.431 \\
\hline
\end{tabular}

NA - data not analyzed.

$p$ values calculated using independent sample $t$ test, Mann-Whitney $U$-test or Chi-2 test

${ }^{\mathrm{a}}$ Normal kidney function (CKD-stages $\left.1-2\right), \mathrm{n}=52$ at 75 and 85 years; poor kidney function (CKD-stages $3 \mathrm{~b}-5$ ), $\mathrm{n}=26$ at 75 and 85 years

${ }^{\mathrm{b}}$ Median with interquartile range

stable as they aged. A poor compared to a normal kidney function over time (from ages 75-85) was associated with higher weight and higher levels of markers of bone metabolism. Furthermore, mean PTH increased over time in women who maintained CKD stages $3 \mathrm{~b}-5(n=26)$ over 10 years. Although these results are based on a very small sample, this increase (despite a relatively stable eGFR) might represent a resistance to the activity of $\mathrm{PTH}$, as previously described [5].

\section{Limitations}

Firstly, we acknowledge that participants might be healthier than women who declined to participate [42], although the random population-based sampling with a baseline attendance rate of $67 \%$, which is comparatively very high, to an extent, reduces this. By the same token it might attract participants with an interest in osteoporosis and fracture. This limitation also applies to follow-up as the number is reduced from either mortality, indications of declining health or for various other reasons. In this study, this is mirrored in the eGFR values differing between attendees and non-attendees although, as previously reported not significantly between ages 75 and 80 $(p=0.123)$ but between ages 80 and $85(p=0.047)$ with eGFR as a predictor of mortality [6]. This is an inherent problem in all longitudinal studies of the elderly, which we have addressed by presenting both cross-sectional and longitudinal data. In addition, in an attempt to control for this, we have specifically evaluated those surviving, comparing those with good and poor function. Albeit, data needs to be interpreted with caution due to smaller numbers at the final follow-up, few other studies of same-aged women have been able to provide a similar analysis.

Secondly, the definition of normal kidney function in the "normal" older person is not without reservation since CKD staging may not fully apply. Reduced kidney function is a common state in the elderly, and many individuals in stage $3 \mathrm{a}$ without proteinuria might never reach stages $3 b-5$. We lack direct measurement, and data on urine protein would have been an advantage in this cohort. It is, however, plausible that cysC estimates of kidney function are more accurate compared to the 
creatinine-based equations used in most previous studies of older people.

Thirdly, due to the observational study design, we cannot draw any conclusions about causality, but the 10-year followup and the absence of exclusion criteria enable reliable conclusions regarding skeletal changes over time that are generalizable to Caucasian women of old age. Importantly, the results are not substantially confounded by anti-osteoporotic medications. Hence, the study addresses the gap apparent from the literature review.

In summary, kidney function is positively associated with FN BMD in elderly women, although the association attenuates as aging progresses. A reduction of kidney function is furthermore associated with bone loss and affected mineral homeostasis. We therefore conclude that reduced kidney function is harmful to bone mass in older women.

\begin{abstract}
Acknowledgements Thanks are extended to the funders, the research nurses and data management at the Clinical and Molecular Osteoporosis Research Unit, Malmö, and to all the women who kindly participated in the study. Thanks are also extended to Paul Gerdhem and Karl Obrant for data collection and to Jan-Åke Nilsson for statistical advice. This work was supported by grants from the Swedish Research Council (K201552X-14691-13-4), Forte (Grant 2007-2125), Greta and Johan Kock Foundation, A. Påhlsson Foundation, A. Osterlund Foundation, H Järnhardt Foundation, King Gustav V 80-year fund, King Gustav V and Queen Victoria Foundation, Åke Wiberg Foundation, Thelma Zoegas Foundation, the Swedish Kidney Foundation, Njurstiftelsen, the Royal Physiographic Society of Lund, Skåne University Hospital Research Fund and the Research and Development Council of Region Skåne, Sweden.
\end{abstract}

\section{Compliance with ethical standards}

\section{Conflicts of interest None.}

Ethical approval The study was approved by the Regional Ethical Review Board in Lund and performed in accordance with the Helsinki declaration.

\section{Informed consent Participants provided written informed consent.}

Open Access This article is distributed under the terms of the Creative Commons Attribution-NonCommercial 4.0 International License (http:// creativecommons.org/licenses/by-nc/4.0/), which permits any noncommercial use, distribution, and reproduction in any medium, provided you give appropriate credit to the original author(s) and the source, provide a link to the Creative Commons license, and indicate if changes were made.

\section{References}

1. Kinsella K, He W (2009) An aging world: 2008. International Population Reports, P95/09-1, U.S. Census Bureau. U.S. Government Printing Office, Washington, DC

2. Lindeman RD, Tobin JD, Shock NW (1984) Association between blood pressure and the rate of decline in renal function with age. Kidney Int 26(6):861-868
3. Kanis JA, Johnell O, Oden A, Sembo I, Redlund-Johnell I, Dawson A, De Laet C, Jonsson B (2000) Long-term risk of osteoporotic fracture in Malmo. Osteoporos Int 11(8):669-674

4. KDIGO (2012) Clinical practice guideline for the evaluation and management of chronic kidney disease (2013). Kidney Int Suppl 3: $19-62$

5. KDIGO (2009) Clinical practice guideline for the diagnosis, evaluation, prevention, and treatment of Chronic Kidney DiseaseMineral and Bone Disorder (CKD-MBD). Kidney Int Suppl 113(3):19-62. https://doi.org/10.1038/ki.2009.188

6. Malmgren L, McGuigan FE, Berglundh S, Westman K, Christensson A, Akesson K (2015) Declining estimated glomerular filtration rate and its association with mortality and comorbidity over 10 years in elderly women. Nephron 130(4):245-255. https://doi.org/10.1159/000435790

7. Inker LA, Schmid CH, Tighiouart H, Eckfeldt JH, Feldman HI, Greene T, Kusek JW, Manzi J, Van Lente F, Zhang YL, Coresh J, Levey AS (2012) Estimating glomerular filtration rate from serum creatinine and cystatin C. N Engl J Med 367(1):20-29. https://doi. org/10.1056/NEJMoa1114248

8. Stevens LA, Schmid CH, Greene T, Li L, Beck GJ, Joffe MM, Froissart M, Kusek JW, Zhang YL, Coresh J, Levey AS (2009) Factors other than glomerular filtration rate affect serum cystatin C levels. Kidney Int 75(6):652-660. https://doi.org/10.1038/ki. 2008.638

9. Fliser D, Ritz E (2001) Serum cystatin C concentration as a marker of renal dysfunction in the elderly. Am J Kidney Dis 37(1):79-83

10. Shlipak MG, Sarnak MJ, Katz R, Fried LF, Seliger SL, Newman AB, Siscovick DS, Stehman-Breen C (2005) Cystatin C and the risk of death and cardiovascular events among elderly persons. N Engl J Med 352(20):2049-2060. https://doi.org/10.1056/NEJMoa043161

11. Gerdhem P, Ivaska KK, Alatalo SL, Halleen JM, Hellman J, Isaksson A, Pettersson K, Vaananen HK, Akesson K, Obrant KJ (2004) Biochemical markers of bone metabolism and prediction of fracture in elderly women. J Bone Miner Res 19(3):386-393. https://doi.org/10.1359/jbmr.0301244

12. Lenora J, Akesson K, Gerdhem P (2010) Effect of precision on longitudinal follow-up of bone mineral density measurements in elderly women and men. J Clin Densitom 13(4):407-412. https:// doi.org/10.1016/j.jocd.2010.04.004

13. Buchebner D, Malmgren L, Christensson A, McGuigan F, Gerdhem P, Ridderstråle M, Åkesson K (2017) Longitudinal assessment of PTH in community-dwelling older women-elevations are not associated with mortality. J Endocr Soc 1(6):615624. https://doi.org/10.1210/js.2017-00104

14. Ivaska KK, Gerdhem P, Vaananen HK, Akesson K, Obrant KJ (2010) Bone turnover markers and prediction of fracture: a prospective follow-up study of 1040 elderly women for a mean of 9 years. J Bone Miner Res 25(2):393-403. https://doi.org/10.1359/jbmr. 091006

15. Gerdhem P, Obrant KJ (2004) Bone mineral density in old age: the influence of age at menarche and menopause. J Bone Miner Metab 22(4):372-375. https://doi.org/10.1007/s00774-004-0497-z

16. Aggarwal HK, Jain D, Yadav S, Kaverappa V (2013) Bone mineral density in patients with predialysis chronic kidney disease. Ren Fail 35(8):1105-1111. https://doi.org/10.3109/0886022X.2013.815102

17. Buchanan JR, Myers CA, Greer RB 3rd (1988) Effect of declining renal function on bone density in aging women. Calcif Tissue Int 43(1):1-6

18. Choi SW, Kim HY, Ahn HR, Lee YH, Kweon SS, Choi JS, Rhee JA, Nam HS, Jeong SK, Park KS, Ryu SY, Song HR, Shin MH (2013) Association of bone mineral density with albuminuria and estimated glomerular filtration rate: the Dong-gu study. Kidney Blood Press Res 37(2-3):132-141. https://doi.org/10.1159/ 000350067 
19. Hsu CY, Cummings SR, McCulloch CE, Chertow GM (2002) Bone mineral density is not diminished by mild to moderate chronic renal insufficiency. Kidney Int 61(5):1814-1820. https://doi.org/10. 1046/j.1523-1755.2002.00306.x

20. Jassal SK, von Muhlen D, Barrett-Connor E (2007) Measures of renal function, BMD, bone loss, and osteoporotic fracture in older adults: the Rancho Bernardo study. J Bone Miner Res 22(2):203210. https://doi.org/10.1359/jbmr.061014

21. Jung YS, Hwang HJ, Yun BH, Chon SJ, Cho S, Choi YS, Kim YT, Lee BS, Seo SK (2014) Renal function is associated with bone mineral density and arterial stiffness in healthy postmenopausal women. Gynecol Obstet Investig 78(2):124-129. https://doi.org/ 10.1159/000363746

22. Kaji H, Yamauchi M, Yamaguchi T, Shigematsu T, Sugimoto T (2010) Mild renal dysfunction is a risk factor for a decrease in bone mineral density and vertebral fractures in Japanese postmenopausal women. J Clin Endocrinol Metab 95(10):4635-4642. https://doi. org/10.1210/jc.2010-0099

23. Kim HL, Park IY, Choi JM, Hwang SM, Kim HS, Lim JS, Kim M, Son MJ (2011) A decline in renal function is associated with loss of bone mass in Korean postmenopausal women with mild renal dysfunction. J Korean Med Sci 26(3):392-398. https://doi.org/10. 3346/jkms.2011.26.3.392

24. Kinsella S, Chavrimootoo S, Molloy MG, Eustace JA (2010) Moderate chronic kidney disease in women is associated with fracture occurrence independently of osteoporosis. Nephron Clin Pract 116(3):c256-c262. https://doi.org/10.1159/000317207

25. Lee YH, Kim JE, Roh YH, Choi HR, Rhee Y, Kang DR, Lim SK (2014) The combination of vitamin D deficiency and mild to moderate chronic kidney disease is associated with low bone mineral density and deteriorated femoral microarchitecture: results from the KNHANES 2008-2011. J Clin Endocrinol Metab 99(10):38793888. https://doi.org/10.1210/jc.2013-3764

26. Lobao R, Carvalho AB, Cuppari L, Ventura R, Lazaretti-Castro M, Jorgetti V, Vieira JG, Cendoroglo M, Draibe SA (2004) High prevalence of low bone mineral density in pre-dialysis chronic kidney disease patients: bone histomorphometric analysis. Clin Nephrol 62(6):432-439

27. Manghat P, Fraser WD, Wierzbicki AS, Fogelman I, Goldsmith DJ, Hampson G (2010) Fibroblast growth factor-23 is associated with C-reactive protein, serum phosphate and bone mineral density in chronic kidney disease. Osteoporos Int 21(11):1853-1861. https:// doi.org/10.1007/s00198-009-1142-4

28. Myong JP, Kim HR, Koo JW, Park CY (2013) Relationship between bone mineral density and moderate to severe chronic kidney disease among general population in Korea. J Korean Med Sci 28(4):569-574. https://doi.org/10.3346/jkms.2013.28.4.569

29. Nickolas TL, Stein EM, Dworakowski E, Nishiyama KK, Komandah-Kosseh M, Zhang CA, McMahon DJ, Liu XS, Boutroy S, Cremers S, Shane E (2013) Rapid cortical bone loss in patients with chronic kidney disease. J Bone Miner Res 28(8): 1811-1820. https://doi.org/10.1002/jbmr.1916

30. Obatake N, Ishimura E, Tsuchida T, Hirowatari K, Naka H, Imanishi Y, Miki T, Inaba M, Nishizawa Y (2007) Annual change in bone mineral density in predialysis patients with chronic renal failure: significance of a decrease in serum 1,25-dihydroxy-vitamin
D. J Bone Miner Metab 25(1):74-79. https://doi.org/10.1007/ s00774-006-0730-z

31. Rix M, Andreassen H, Eskildsen P, Langdahl B, Olgaard K (1999) Bone mineral density and biochemical markers of bone turnover in patients with predialysis chronic renal failure. Kidney Int 56(3): 1084-1093. https://doi.org/10.1046/j.1523-1755.1999.00617.x

32. Shin JH, Kim SH, Yu SH (2014) Metabolic syndrome and chronic kidney disease as risk factors of osteoporosis. Clin Nephrol 81(1): $1-8$

33. Tomida K, Hamano T, Mikami S, Fujii N, Okada N, Matsui I, Nagasawa Y, Moriyama T, Ito T, Imai E, Isaka Y, Rakugi $\mathrm{H}$ (2009) Serum 25-hydroxyvitamin D as an independent determinant of 1-84 PTH and bone mineral density in non-diabetic predialysis CKD patients. Bone 44(4):678-683. https://doi.org/10.1016/j.bone. 2008.11.016

34. Tseng T, Mu C, Hsu C (2014) The correlation between renal function and bone mineral density. Minerva Urol Nefrol 66(3):153-156

35. Yenchek RH, Ix JH, Shlipak MG, Bauer DC, Rianon NJ, Kritchevsky SB, Harris TB, Newman AB, Cauley JA, Fried LF, Health, Aging, and Body Composition Study (2012) Bone mineral density and fracture risk in older individuals with CKD. Clin J Am Soc Nephrol 7(7):1130-1136. https://doi.org/10.2215/CJN 12871211

36. Christensson A, Elmstahl S (2011) Estimation of the age-dependent decline of glomerular filtration rate from formulas based on creatinine and cystatin $\mathrm{C}$ in the general elderly population. Nephron Clin Pract 117(1):c40-c50. https://doi.org/10.1159/000319646

37. El Maghraoui A, Sadni S, El Maataoui A, Majjad A, Rezqi A, Ouzzif Z, Mounach A (2015) Influence of obesity on vertebral fracture prevalence and vitamin D status in postmenopausal women. Nutr Metab 12:44. https://doi.org/10.1186/s12986-015-0041-2

38. Armstrong ME, Spencer EA, Cairns BJ, Banks E, Pirie K, Green J, Wright FL, Reeves GK, Beral V (2011) Body mass index and physical activity in relation to the incidence of hip fracture in postmenopausal women. J Bone Miner Res 26(6):1330-1338. https:// doi.org/10.1002/jbmr.315

39. Anderson JL, Vanwoerkom RC, Horne BD, Bair TL, May HT, Lappe DL, Muhlestein JB (2011) Parathyroid hormone, vitamin $\mathrm{D}$, renal dysfunction, and cardiovascular disease: dependent or independent risk factors? Am Heart J 162(2):331-339 e332. https:// doi.org/10.1016/j.ahj.2011.05.005

40. Sambrook PN, Chen JS, March LM, Cameron ID, Cumming RG, Lord SR, Schwarz J, Seibel MJ (2004) Serum parathyroid hormone is associated with increased mortality independent of 25-hydroxy vitamin $\mathrm{d}$ status, bone mass, and renal function in the frail and very old: a cohort study. J Clin Endocrinol Metab 89(11):5477-5481. https://doi.org/10.1210/jc.2004-0307

41. Levin A, Bakris GL, Molitch M, Smulders M, Tian J, Williams LA, Andress DL (2007) Prevalence of abnormal serum vitamin D, PTH, calcium, and phosphorus in patients with chronic kidney disease: results of the study to evaluate early kidney disease. Kidney Int 71(1):31-38. https://doi.org/10.1038/sj.ki.5002009

42. Wihlborg A, Akesson K, Gerdhem P (2014) External validity of a population-based study on osteoporosis and fracture. Acta Orthop 85(4):433-437. https://doi.org/10.3109/17453674.2014.920987 